\title{
EFFECT OF BEHAVIORAL PROBLEMS OF CHILDREN WITH AUTISM ON THEIR MOTHER'S QUALITY OF LIFE
}

\author{
Ayesha Waheed, Mussarat Jabeen Khan*, Maryam Khurshid* \\ Foundation University, Rawalpindi Pakistan, *International Islamic University, Islamabad Pakistan
}

\begin{abstract}
Objective: To assess the effect of behavioral problems of children with Autism on their mother's quality of life. Study Design: Cross-sectional study design.

Place and Duration of Study: The sample was selected from different special education institutes of Islamabad and Rawalpindi, from Mar 2017 to Sep 2017.

Methodology: The study was conducted on 100 participants $(n=50$ mothers, $n=50$ diagnosed children with Autism). Purposive sampling technique was used to collect data. World Health Organization Quality of life scaleBREF and Childhood Autism Rating Scale-CARS were used.

Results: Results showed that children's autism related behavioral problem has a significant negative relationship with quality of life of their mothers $(B=-0.67, p<0.05)$. Quality of life was found high in postgraduate mothers, $\mathrm{n}=25(50 \%)$ while autism related behavioral problems were less in children with postgraduate mothers. Results of t-test analysis showed that mothers living in joint family scored high on quality of life. Quality of life among working mothers was found high and children of working mothers exhibited less autism related behavioral problems than children of non-working mothers as indicated by t-test analysis.

Conclusion: Present study demonstrated that children's autism related behavioral problems were negatively related with their mother's quality of life. Postgraduate, working mothers living in joint family system have better quality of life than undergraduate, non-working mothers living in nuclear family system.
\end{abstract}

Keywords: Autism, Behavioral problems, Quality of life.

This is an Open Access article distributed under the terms of the Creative Commons Attribution License (http://creativecommons.org/licenses/by/4.0), which permits unrestricted use, distribution, and reproduction in any medium, provided the original work is properly cited.

\section{INTRODUCTION}

Autism is a behaviorally-defined condition but is caused by several different known and unknown biologically based brain dysfunctions that affect the developing brain's ability to handle information ${ }^{1}$. The challenges a family and especially a mother faces as being a parent of children with Autism Spectrum Disorder (ASD) begin with the diagnosis of the disorder. Accepting this reality brings anxiety, stress and negative thinking of life been unfair to her and then the thinking that its lifelong responsibility on her makes it more stressful. The other factor is financial burden as well as physical effort to fulfil the extensive needs of the child with ASD ${ }^{2,3}$. An important aspect of one's life is its quality, which is measured by assessing physical and mental health, along with the social and environmental

Correspondence: Dr Mussarat Jabeen Khan, Department of Psychology, International Islamic University, Islamabad Pakistan Received: 23 Oct 2019; revised received: 27 Dec 2019; accepted: 01 Dec 2019 relationships. Quality of life of mothers with Child with Autism is affected by the responsibilities for caring of special needs of their children in addition to the expected roles 4,5 . The stress of living with a child with Autism can affect the overall psychological wellbeing of a family as well as generates conflicts and upsets among them ${ }^{6}$.

It is established that both parents are involved in a child's upbringing and are supposed to share the responsibility. But on the other side, it is a culturally established fact that mother is always on a sharp edge as she gives birth, feeds the baby and helps him achieve developmental milestones. As the mother spends more time with her child, her life basically revolves around him. Every single emotion of child brings the same emotion in mother. When the child is comfortable, mother is relaxed, if the child is upset, so is the mother. She is the one who is believed to educate her child, teach good manners, inculcate appropriate 
character, and bring good out of him. Every good or inappropriate behavior exhibited by the child is targeted towards the medium of upbringing displayed by the mother. Keeping in mind this social allegation, history also witnesses such kind of societal treatment towards mother?

Behavior problems in child with Autism become a concerned reason for parents, especially mother's stress. Various studies have shown the same relationship between maternal stress and children's maladaptive behaviors ${ }^{8,9}$. It was also found that parents especially mothers of children with developmental disabilities experienced heightened stress ${ }^{10}$.

Objective of the current study was to explore the effect of Autism related behavioral problems of children on their mother's quality of life. The equilibrium of a family disturbs when they find their child in a condition like autism. In such cases, mothers often seem to negatively compromise over their standards and self-worth, which leads to a lower quality of life. In this study, behavioral problems of children with Autism and their effects in the quality of life of their mothers and the role of some demographic variables such as family system, education and occupation of those mothers were studied.

\section{METHODOLOGY}

In this cross-sectional study purposive sampling techniques were employed to collect data. Sample size was calculated by using the WHO sample size calculator, considering 5\% margin of error and a 95\% confidence interval. Sample comprised of 50 diagnosed Children with Autism and their mothers taken from different special education institutes of Islamabad and Rawalpindi. Nearly 16 per 1,000 children between 3 and 9 years of age suffer from severe mental retardation. Pakistani children had a slightly increased prevalence of autism (2.57/ $1,000)^{11}$. Inclusion criteria were only those children who were having autistic features and their mothers having 10 years of education. Exclusion criteria were those having any other physiological problem. Children with Autism with age range of 5-12 years were selected. Sample was selected from different special schools of Islamabad and Rawalpindi, able to read and understand Urdu.

World Health Organization Quality of Life Scale (WHOQOL-BREF) was used to measure quality of life ${ }^{11}$. It comprises of 26 items. The Childhood Autism Rating Scale (CARS) was used to identify children with Autism and to distinguish them from other developmentally delayed children. It is comprised of 15 items $^{12}, 13$, and is also able to distinguish among children with mild to moderate and moderate to severe Autism.

The authorities of different special schools of Islamabad and Rawalpindi were approached for data collection after getting approval from Department of Psychology Ethics Committee (DPEC) with IERB Number-20DPEC. Children and their mothers were approached individually, and the consent of participation was taken from them. They were assured that all the information will be kept confidential and will only be used for research purpose. In addition to the consent form and demographic sheet, World Health Organization Quality of Life Scale (WHOQOL-BREF) was given to mothers and they were briefed about how to respond to the questionnaire. Rating on Childhood Autism Rating Scale (CARS) was taken by the teachers of special education schools, who were involved in teaching with these children. Respondents were asked to read each item of the scale carefully and appropriately mark the answers. All their misapprehensions about the study were cleared before and after the administration of questionnaire. Data was analyzed through SPSS (version 21). Different statistics were applied like Descriptive statistics were used to define demographic variables. Student t-test analysis was used to examine the differences between groups as postgraduate and undergraduate mothers, working and non-working mothers, nuclear family system and joint family system. Linear regression analysis was used to investigate the effect of behavioral problems of children with autism on their mother's quality of life. 


\section{RESULTS}

There were 28 (56\%) boys and $22(54 \%)$ girls with autism. About 25 (50\%) mothers were undergraduates and $25(50 \%)$ were postgraduate. These were $25(50 \%)$ mothers working and 25 (50\%) non-working mothers. About 29 (58\%) mothers from nuclear family system and 21 (52\%) behavioral problems $(\mathrm{M}=38.60 \pm 4.64)$ than children of undergraduate mothers $(M=40.55 \pm 5.01)$.

Table-II showed the significant differences between working and non-working mothers on their quality of life and childhood autism related behavioral problems. Mean column shows that quality of life of working mothers is better $(\mathrm{M}=$

Table-I: Results between undergraduate and postgraduate mothers on quality of life and childhood autism related scale $(n=50)$.

\begin{tabular}{l|c|c|c|c|c|c}
\hline & $\begin{array}{c}\text { Undergraduate } \\
(\mathbf{n = 2 5 )}\end{array}$ & $\begin{array}{c}\text { Postgraduate } \\
(\mathbf{n = 2 5 )}\end{array}$ & \multirow{2}{*}{$\boldsymbol{p}$-value } & \multicolumn{2}{|c|}{$\mathbf{9 5 \% \text { Class Interval }}$} & \multirow{2}{*}{ Cohen's d } \\
\cline { 5 - 6 } & Mean \pm SD) & Mean \pm SD & & Lower Limit & Upper Limit & \\
\hline Variables & $52.91 \pm 7.33$ & $53.82 \pm 11.17$ & 0.02 & 8.11 & 6.28 & 0.60 \\
\hline Quality of Life & $40.55 \pm 5.01$ & $38.60 \pm 4.64$ & 0.03 & 1.29 & 5.18 & 0.40 \\
\hline $\begin{array}{l}\text { Childhood Autism } \\
\text { Related Scale }\end{array}$ & & &
\end{tabular}

Table-II: Results between working and non-working mothers on quality of life and childhood autism related scale $(n=50)$.

\begin{tabular}{l|c|c|c|c|c|c}
\hline & \multirow{2}{*}{$\begin{array}{c}\text { Working } \\
(\mathbf{n = 2 5})\end{array}$} & \multirow{2}{*}{$\begin{array}{c}\text { Non-working } \\
(\mathbf{n = 2 5 )}\end{array}$} & \multirow{2}{*}{$\begin{array}{c}\boldsymbol{p} \text { - } \\
\text { value }\end{array}$} & \multicolumn{2}{|c|}{$\mathbf{9 5 \% \text { Class Interval }}$} & \multirow{2}{*}{ Cohen's d } \\
\cline { 5 - 6 } & Mean \pm SD & Mean \pm SD & & Lower Limit & Upper Limit & \\
\hline Variables & $54.44 \pm 8.72$ & $52.80 \pm 11.95$ & 0.03 & -7.59 & -4.31 & 0.84 \\
\hline Quality of Life & $34.88 \pm 4.99$ & $39.18 \pm 4.58$ & 0.00 & 3.02 & 2.42 & 0.62 \\
\hline $\begin{array}{l}\text { Childhood Autism } \\
\text { Related Scale }\end{array}$ & & & &
\end{tabular}

Table-III: Results between mothers living in nuclear and joint family systems on quality of life and childhood autism related scale $(n=50)$.

\begin{tabular}{|c|c|c|c|c|c|c|}
\hline & Nuclear $(n=29)$ & Joint $(n=21)$ & \multirow{2}{*}{$\begin{array}{c}p- \\
\text { value }\end{array}$} & \multicolumn{2}{|c|}{ 95\% Class Interval } & \multirow{2}{*}{ Cohen's d } \\
\hline Variables & Mean \pm SD & Mean \pm SD & & Lower Limit & Upper Limit & \\
\hline Quality of Life & $52.28 \pm 9.56$ & $55.48 \pm 11.39$ & 0.02 & -9.17 & -2.77 & 0.80 \\
\hline $\begin{array}{l}\text { Childhood Autism } \\
\text { Related Scale }\end{array}$ & $39.86 \pm 5.48$ & $37.88 \pm 3.28$ & 0.01 & 0.72 & 4.68 & 0.20 \\
\hline
\end{tabular}

Table-IV: Linear regression analysis showing effect of childhood autism related behavioral problems on mother's quality of life $(n=50)$.

\begin{tabular}{l|c|c|c|c|c}
\hline Model & B & Standard Error & B & t & $p$-value \\
\hline Constant & 40.79 & 3.59 & & 11.35 & 0.00 \\
\hline Children with Autism & -0.03 & 0.06 & -0.67 & 0.49 & 0.02 \\
\hline R2 $=0.25$ & & & & & \\
\hline
\end{tabular}

from joint family system. Mean age of children $10.50 \pm 0.50$ years whereas mean age of mothers was $45 \pm 2.50$ years.

Table-I showed the significant differences between postgraduate and undergraduate mothers on their quality of life and childhood autism related behavioral problems. Mean column shows that quality of life of postgraduate mothers is better $(\mathrm{M}=53.82 \pm 11.17)$ than undergraduate mothers $(\mathrm{M}=52.91 \pm 7.33)$. Whereas children of postgraduate mothers have less autism related
$54.44 \pm 8.72)$ than non-working mothers $(\mathrm{M}=52.80$ \pm 11.95). Whereas children of working mothers have less autism related behavioral problems $(\mathrm{M}=34.88 \pm 4.99)$ than children of non-working mothers $(\mathrm{M}=39.18 \pm 4.58)$.

Table-III showed the significant differences between mothers living in nuclear and joint family system on their quality of life and childhood autism related behavioral problems. Mean column shows that quality of life of mothers living in joint family system is better $(\mathrm{M}=55.48 \pm$ 
11.39) than mothers living in nuclear family system $(\mathrm{M}=52.28 \pm 9.56)$. Whereas children belonging to joint family system have less autism related behavioral problems $(\mathrm{M}=37.88 \pm 3.28)$ than children living in nuclear family system (M $=39.86 \pm 5.48$ ).

Table-IV showed that childhood autism related behavioral problems predict mother's quality of life. The R2 value of 0.25 indicated that $25 \%$ variance in the dependent variable which was mother's quality of life can be accounted for by the predictor which is childhood autism related behavioral problems with $\mathrm{F}=0.25, p<0.05$. Childhood autism related behavioral problems has a significant negative effect on Mother's quality of life $(\beta=-0.67, p<0.05)$.

\section{DISCUSSION}

The present study was intended to investigate the effect of behavioral problems of children with Autism on Quality of life of their mothers. Autism is a neurological disorder that dominates often in terms of behavioral issues. Families with an autistic child usually suffer from a great deal of stress as their social and emotional inter relations are compromised and limited. Particularly talking about mothers, they seem to have more stress. In Pakistani culture, there are many superstitious beliefs that prevail till now. One of them is that mother is usually responsible for the deformities and abnormalities of their children. They often face accusations like that from their families and society around them. Such beliefs of a society place the mothers with special child under a great deal of stress which in turn affect their quality of life ${ }^{14}$.

Quality of life of a person is dependent on various factors among which if one or two are compromised, may affect the whole personality. Behavioral problems of children with Autism may restrict their mother's social and familial activities which are often arranged for ones better physical and psychological health. If a mother with a child with Autism has limited access to such events, she may have a compromised quality of life. Riany, Cuskelly, and Meredith ${ }^{15}$, has highlighted in their study that behavioral problems of a child with Autism were often associated with mother's higher stress level. The goal of this study was to investigate how Indonesian mothers from a range of backgrounds and without a child with autism understood autism and the most appropriate ways to parent such a child. The study was a qualitative investigation using semi-structured interviews with nine Indonesian mothers. Five aspects were investigated: understanding about autism, beliefs about causes of autism, possible reactions to having a child with autism, perceptions of parenting a child with autism, and perceptions of parent-child relationships.

Results have shown a significant difference among the anticipated variables. A research conducted by Emerson ${ }^{16}$, showed that mothers with an intellectually disabled child like Autism, face financial constraints and were economically disadvantaged, offered less social support, have increased stress and were more likely to live in an unhealthy family, and so, their psychological wellbeing is adverse. Another study conducted by Vasilopoulou and Nisbet 17 , indicated few factors led to lower maternal Quality of life among which unemployment was one. As a working mother can easily manage the financial constraints resulting from the special needs of a child with Autism, she will face less stress and hence her Quality of life will be better.

The study findings showed there has been a noticeable difference in the quality of life of mothers of Autistic children with high education as compared to those with low education. Ross and Van Willigen ${ }^{18}$, proposed that education affects an individual's subjective quality of life by contributing towards person's wellbeing. It was noticed that mothers of Autistic children with high education exhibited flexibility towards the adaptation of family norms and life style. Such resilience contributed towards their wellbeing and better quality of life. Education also enabled them to be aware of and get better understanding of their child's problem and learn new ways of handling the issues generated by the child's 
behavior problems affecting child as well as the family.

In Pakistan, joint family system is the primary form of familial institution. Grandparents are considered as vital emotional support for other family members ${ }^{19}$. Gray 20 found in his study that parents, especially mothers of Autistic children tend to seek more social support. This social support when available in extended family can prove to be a declining factor for stress as suggested by Gallagher and Vella-Brodrick ${ }^{21}$, that social support was inversely related to the distress and anxiety in parents with intellectually disabled children. As mothers living in joint families get the required emotional support, which is less available in nuclear family system, they tend to have a better quality of life.

The present study was, although a significant contribution in the indigenous literature concerning Autism and its management, however, like every study the present one also has some shortcomings. The present study was conducted with most available sample yet, new facts could be learned in future studies by increasing sample size. Since the study was conducted in relatively developed region of Pakistan, where middle and upper middle socioeconomic status is predominantly prevalent, while lower socioeconomic status can reveal important findings related to existence and management of autistic symptoms.

\section{CONCLUSION}

Behavioral problems of children with Autism were negatively correlated to quality of life of their mothers. Children of postgraduate, working mothers had less autism related behavioral problems than children of undergraduate, non-working mothers. Children belonging to joint family system had less autism related behavioral problems than children living in nuclear family system.

\section{CONFLICT OF INTEREST}

This study has no conflict of interest to be declared by any authors.

\section{REFERENCES}

1. Conner CM, White SW. Stress in mothers of children with autism: Trait mindfulness as a protective factor. Res Autism Spect Disord 2014; 8(6): 617-24

2. Carrington SJ, Kent RG, Maljaars J, Le Couteur A, Gould J, Wing L, et al. DSM-5 Autism Spectrum Disorder: In search of essential behaviours for diagnosis. Res Autism Spect Disord 2014; 8(6): 701-15.

3. Estes A, Munson J, Rogers SJ, Greenson J, Winter J, Dawson G. LongTerm outcomes of early intervention in 6-year-old children with autism spectrum disorder. J Am Acad Child Adolesc Psy 2015; 54(7): $580-87$.

4. Schertz HH, Odom SL, Baggett KM, Sideris JH. Mediating parent learning to promote social communication for toddlers with autism: effects from a randomized controlled trial. J Autism Dev Disord 2018; 48(3): 853-67.

5. Hartley SL, Schultz HM. Support needs of fathers and mothers of children and adolescents with autism spectrum disorder. J Autism Dev Disord 2015; 45(6): 1636-48.

6. Schlebusch L, Dada S, Samuels AE. Family quality of life of South African families raising children with autism spectrum disorder. J Autism Dev Disord 2017; 47(7): 1966-77.

7. Soltanifar A, Akbarzadeh F, Moharreri F, Soltanifar A, Ebrahimi A, Mokhber N. Comparison of parental stress among mothers and fathers of children with autistic spectrum disorder in İran. Iran J Nurs Midwifery Res 2015; 20(1): 93-98.

8. Baxter AJ, Brugha TS, Erskine HE. The epidemiology and global burden of autism spectrum disorders. Psy Med 2015; 45(3): 601-13.

9. Wang F, Lu L, Wang SB. The prevalence of autism spectrum disorders in China: a comprehensive meta-analysis. Int J Biol Sci 2018; 14(7): 717-25.

10. Krakovich TM, Mc Grew JH, Yu Y. Stress in parents of children with autism spectrum disorder: an exploration of demands and resources. J Autism Dev Disord 2016; 46(6): 2042-53.

11. Junior SBM, Celestino MIO, Serra JPC. Risk and protective factors for symptoms of anxiety and depression in parents of children with autism spectrum disorder. Dev Neurorehabilit. 2016; 19(3): 146-53.

12. Huang $\mathrm{CY}$, Yen HC, Tseng MH. Impacts of autistic behaviors, emotional and behavioral problems on parenting stress in caregivers of children with autism. J Autism Dev Disord 2014; 44(6): 1383-90.

13. Epstein T, Saltzman-Benaiah J, O'Hare A, Goll JC, Tuck S. Associated features of aspergers syndrome and their relationship to parenting stress. Child: Care, Health Dev 2008; 34 (4): 503-11.

14. Bagner DM, Eyberg SM. Parent-child interaction therapy for disruptive behavior in children with mental retardation: A randomized controlled trial. J Clin Child Adolesc Psychol 2007; 36(3): 418-29.

15. Riany YE, Cuskelly M, Meredith P. Cultural Beliefs about Autism in Indonesia. Int J Dis Dev Edu 2016; 63(6): 623-40.

16. Emerson E. Mothers of children and adolescents with intellec-tual disability: social and economic situation, mental health status, and the self assessed social and psychological impact of the child's difficulties. J Int Disabil 2003; 47(4-5): 385-99.

17. Vasilopoulou E, Nisbet J. The quality of life of parents of children with autism spectrum disorder: A systematic review. Res Autism Spect Disord 2016; 23(1): 36-49.

18. Ross CE, Van Willigen M. Education and the subjective quality of life. J Health Soc Behav 1997; 38(3); 275-97.

19. Kashif A. Joint vs. nuclear family system. Asiana Wedding 2012; 2(6). Available at ayeshaheaven.blogspot.com/2013/07/family-systemjoint-vsnuclear, retrieved on 31.08.2018.

20. Gray DE. Gender and coping: the parents of children with high functioning autism. Soc Sci Med 2006; 56(3): 631-42.

21. Gallagher EN, Vella-Brodrick DA. Social support and emotional intelligence as predictors of subjective well-being. Pers Individ Dif 2008; 44(7): 1551-61. 